\title{
Reverse Engineering Representation Using an Image Processing Modification
}

\author{
Ahmed A. A. Duroobi* \\ Nareen Hafidh Obaeed $* *$ \\ Safaa Kadhim Ghazi**** \\ ***,***Department of Production and Metallurgy Engineering/University of Technology/Baghdad/Iraq \\ *Email: ahmed_abdulsamii7@yahoo.co.uk
}

(Received 6 November 2017; accepted 18 March 2018)

https://doi.org/10.22153/kej.2019.03.001

\begin{abstract}
In the reverse engineering approach, a massive amount of point data is gathered together during data acquisition and this leads to larger file sizes and longer information data handling time. In addition, fitting of surfaces of these data point is time-consuming and demands particular skills. In the present work a method for getting the control points of any profile has been presented. Where, many process for an image modification was explained using Solid Work program, and a parametric equation of the profile that proposed has been derived using Bezier technique with the control points that adopted. Finally, the proposed profile was machined using 3-aixs CNC milling machine and a compression in dimensions process has been occurred between the proposed and original part so as to demonstrate the verification of the proposed method.
\end{abstract}

Keywords: Bezier, reverse engineering, tool path generation.

\section{Introduction}

The progression of a new product is a repeated process, which includes: design of product, product prototyping for experimental assessment and adjustment of design, where computer aided design (CAD) is commonly connected with interactive computer graphics, and the designer can conceptualize the object to designed very easily on the graphics screen and take into account alternative designs or modify a particular design more quickly to meet the needed design requirements or changes of design.

In previous works when the researchers make them design of any profile using MATLAB program they save the extension of the design file as $\mathrm{m}$-file or dxf-file. And then open it in CAM program to get the simulation of machining operation and get G-code. This method can be succeeding for simple surface, but it's useless if complex profile was designed and translated using this basic method. While other researchers write the control points of any complex surface in CAM program manually, and this primitive method also may be taking a long time. Where from MATLAB program it can be made a program to get G-code directly, and this method can be done for 2-axis, 3 -axis at the maximum, while it's difficult to adapt this method to obtain G-code for machining in multi-axis because this need hard work and may be the results that got not compatible with CNC machine control system [1]. Malgozata Ponitowska [2], investigated a method for representing a visual analysis of deviation in 2D graphs in measurements of free-form surface performed by use numerically control CMMs on the basis of a CAD models, the measurement is aim at evaluate the form deviation and thus the greatest deviation of the actual surfaces from the CAD models. Abil Kuş et al [3], reviewed the processes of digitizing a parts and creating a CAD model from 3D scan data use 3D optical scan technology. They illustrated two scanning 
experiments of automotive application, the first one examines the process from scan to remanufacturing the damag sheet metal cutting die, using a 3D scan techniques and the second one compare the scanned points clouds data to 3D CAD data for inspection purpose. Wissam and et al [4], use CMM to measuring external profile of objects with external porosity space. In this processes the center of a CMM probing stylus would be compensate to avoid porosity spaces located above external surfaces area along surfaces normal directions. The proposed method is able to avoid porosity space in CMM measurement regardless of the availability of CAD model. And they present a processed of measure geometrical features of a physical object to convert the measured points cloud into a 3-D models. Dongdong Zhang et al [5], presented an approaching for generating curvature-adaptive finish tool path with bound error directly from massive points data in three-axis computer numerical control (CNC) milling. It enable the generation of curvature-adaptive tool path from massive points data that is critical for balancing the trade-off between machining accuracy and speed. To ensure the path accuracy and robust for arbitrary surface where there might be a sudden curvature change. It overcomes potential excessive locality of curvature-adaptive path by examine the neighboring point curvature within a self-updating search bound. their result affirm that the combination of curvature-adaptive paths generation and the guidance field algorithm produce high-quality numerical control (NC) path from a variety of points cloud data with bound error.

In the present research a new method for getting the control points of any profile will be presented. Where, many process for an image modification will be explained using Solid Work program, and then a parametric equation of the profile that will proposed will be derived using Bezier technique with the control points that adopted.

\section{Construction of Proposed Method}

Actually, the structure of the proposed method in the present research must be divide into four divisions, the first one relate with CAD program (Solid Work), which is deals with the control points that will be detected from an image of the proposed part. Second division relates with derived the parametric equation that describe the profile of the proposed part. Third division represents the CAM field to machine the proposed part. Finally, a comparison process will be occurred to check the verifying of the proposed method.

Reverse engineering is a significant manner in constructing a CAD model from an existing substantial part through part or product design [1]. If it is interested in doing reverse engineering of legacy products, then let me save your time and energy and explain about the best company can outsource reverse engineering of legacy products. Mechanical 3D Modeling can provide complete design support to help you recover design information for your obsolete parts with accuracy. From automotive parts, industrial equipment to entire plants, it has worked on reverse engineering projects of any scale and complexity. It's experienced team and designers utilize Solid Works as well other CAD tools and has remained a valuable partner to many clients for successfully serving reverse engineering projects.

Almost all of the proposed solutions for RE are realized in two proceedings; In the first step, the physical portion is measured or digitized by a measuring instrument such as a coordinate measuring machine (CMM), and surface points are captured in 3D coordinates. While the second step, curves or surfaces be suitable for to the measured points. The absence of coordinate measuring machine, it has been depending on Solid work program in order to obtain the control points of the case study. The getting point has been saved in a way so they can be used in MATLAB program. Also it can trace the procedure in the flowchart as shown figure (1). 


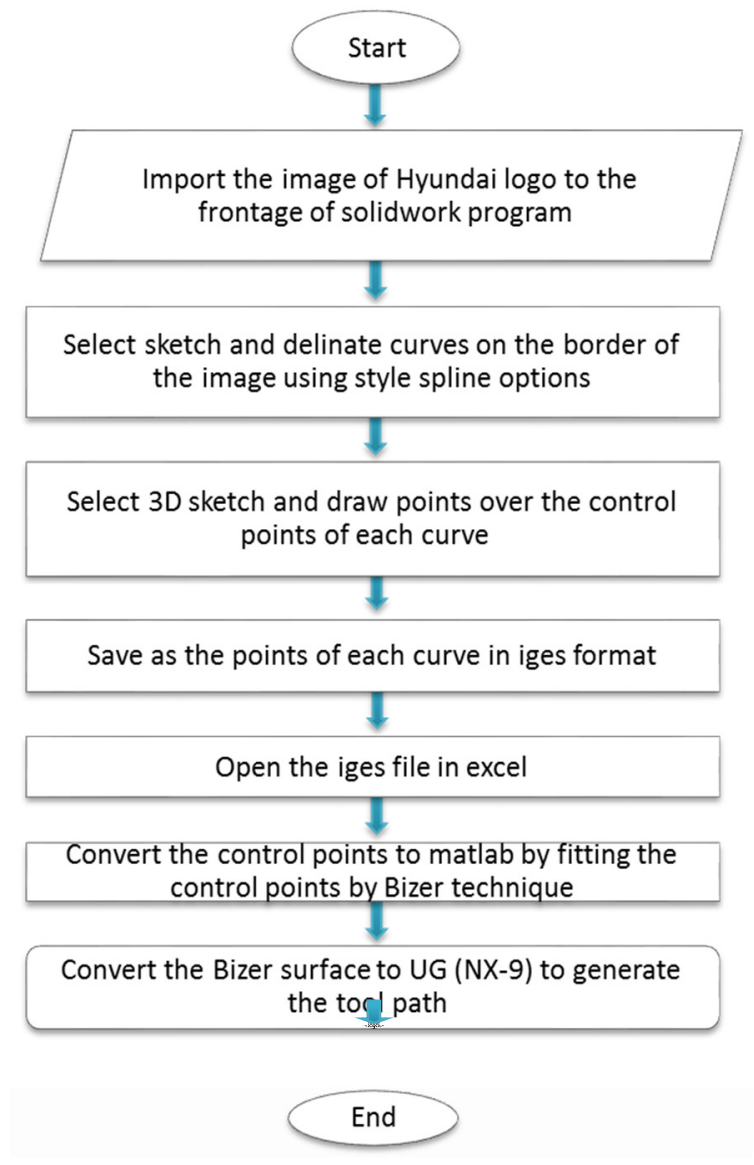

Fig. 1. The flowcharts demonstrate the procedure for recalling the control point using solid work program.

\section{Surface Representation}

In the present research Bezier surface was selected, which is a direct extension of a Bezier curve. The underlying principle in defining a Bezier surface is that to let a point trace out of Bezier curve and then let this curve sweep out a Bezier surface.

The simple extension for three dimensional free-form surfaces from 3-dimensional free-form curve is by incorporating another parameter (w) to the vector equation of the curve to obtain the surface equation [6]:

$P(u, w)=[x(u, w), y(u, w), z(u, w)]$

Where: $0 \leq u, w \leq 1 \quad(u, w)$ are independent variables.

This equation is called bivaraite parametric equation since it includes two variable parameters in two various directions.

The use of higher degree causes small oscillations in curve and requires heavy computations. So in this research Bezier surface with order $n \times m=9 \times 9$ was selected.
By the mathematical derivation, the general equation to represent the Bezier surfaces of higher degrees can be shown as follow [6]:

$P(u, w)=[U][M B][P][M B]^{T}[W]$

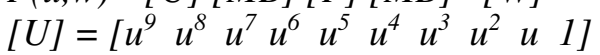

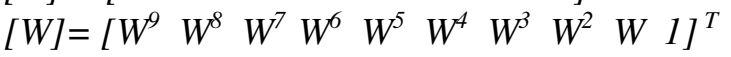

$M_{B}=\left[\begin{array}{cccccccccc}-1 & 9 & -36 & 84 & -126 & 126 & -84 & 36 & -9 & 1 \\ 9 & -72 & 252 & -504 & 630 & -504 & 252 & -72 & 9 & 0 \\ -36 & 252 & -756 & 1260 & -1260 & 756 & -252 & 36 & 0 & 0 \\ 84 & -504 & 1260 & -1680 & 1260 & -504 & 84 & 0 & 0 & 0 \\ -126 & 630 & -1260 & 1260 & -630 & 126 & 0 & 0 & 0 & 0 \\ 126 & -504 & 756 & -504 & 126 & 0 & 0 & 0 & 0 & 0 \\ -84 & 252 & -252 & 84 & 0 & 0 & 0 & 0 & 0 & 0 \\ 36 & -72 & 36 & 0 & 0 & 0 & 0 & 0 & 0 & 0 \\ -9 & 9 & 0 & 0 & 0 & 0 & 0 & 0 & 0 & 0 \\ 1 & 0 & 0 & 0 & 0 & 0 & 0 & 0 & 0 & 0\end{array}\right]$

So, according to these equations above and by select a several control points across $\mathrm{x}, \mathrm{y}$ and $\mathrm{z}-$ axis using MATLAB program, it can be obtain the surface as show figure (2):

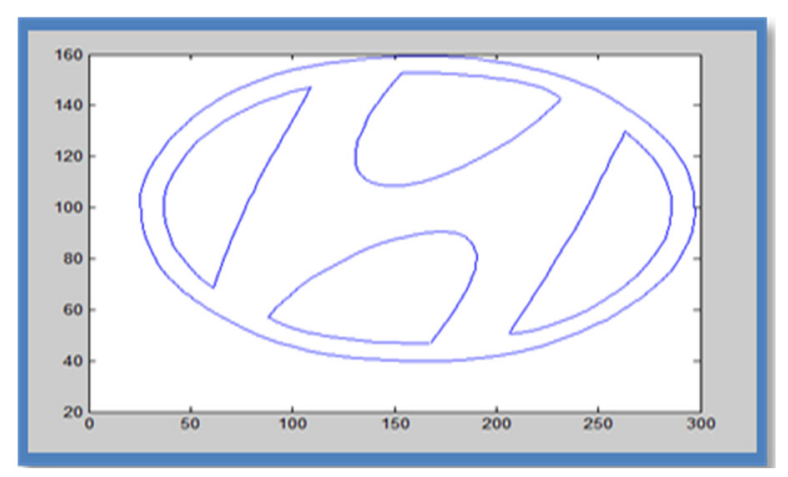

Fig. 2. Represent the higher degree Beziers's Surface in graphical mode.

From the all above, it must be notice that the M-program that was built to obtain the surface in figure (2) should be saved in (dat) extension so as to can open it in UGS program as shown figure (3) to take tool path as shown in figure (4). 


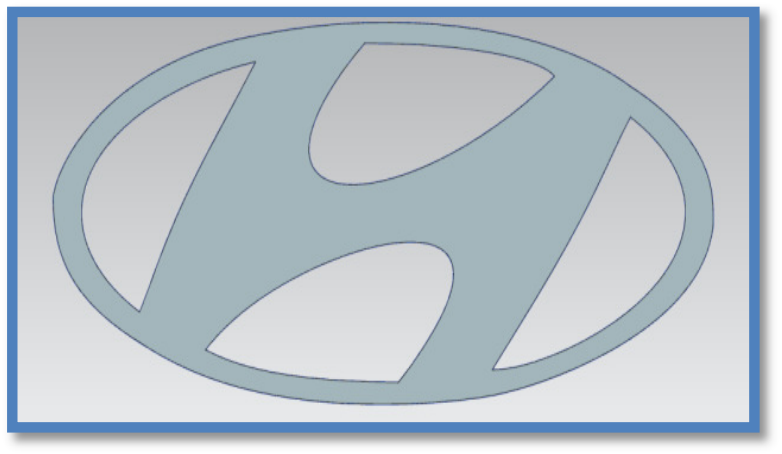

Fig. 3. Model generation using UG-NX program.

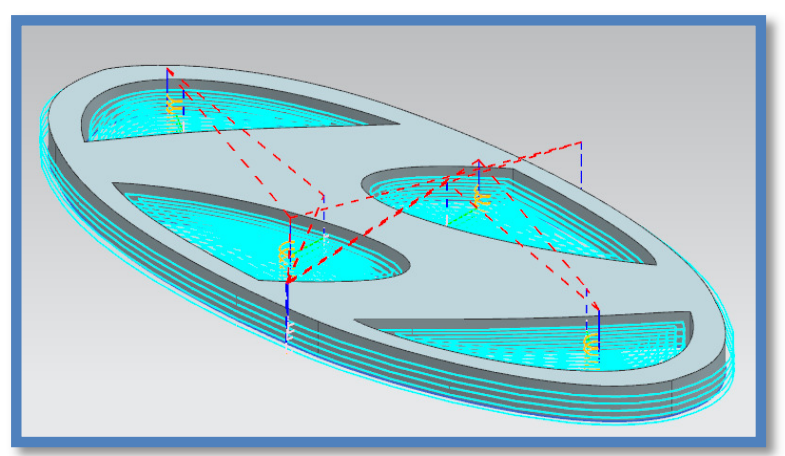

Fig. 4. Tool path using UG-NX program.

\section{Continuity of the Present Work}

To confirm the continuity for suggested work which divide in two zones of the curve based on the Bezier curve $(B=7)$ the kinds for continuity $C$ ${ }^{0}, \mathrm{C}^{1}$ and $\mathrm{C}^{2}$ will be achieve for the first segment of the Bezier curve by blended the region. To confirm the continuity in Bezier, the continuity would utilize to whole the region of the curve so the $\mathrm{Z}$ - axis would constant in value for whole curve of the work like in figure (bellow).

$$
\begin{aligned}
B(u) & =\left[\begin{array}{lllllll}
u^{6} & u^{5} & u^{4} & u^{3} & u^{2} & u & 1
\end{array}\right] \\
B^{\prime}(u) & =\left[\begin{array}{lllllll}
6 u^{5} & 5 u^{4} & 4 u^{3} & 3 u^{2} & 2 u & 1 & 0
\end{array}\right] \\
B^{\prime}(1) & =\left[\begin{array}{llllllll}
1 & 1 & 1 & 1 & 1 & 1 & 0
\end{array}\right] \\
B^{\prime \prime}(u) & =\left[\begin{array}{llllllll}
30 u^{4} & 20 u^{3} & 12 u^{2} & 6 u & 2 & 0 & 0
\end{array}\right] \\
B^{\prime \prime}(1) & =\left[\begin{array}{lllllll}
30 & 20 & 12 & 6 & 2 & 0 & 0
\end{array}\right]
\end{aligned}
$$

By substitute $u=1$ in equation for the first curve and made this equal to equation of the next curve at $(\mathrm{u}=0)$ like equation below.

$\mathrm{B}(\mathrm{u}=$ one $) 1$ first curve $=\mathrm{B}(\mathrm{u}=$ zero $) 2$ next curve to take $\mathrm{C}^{0}$ as shown in figure (5).

Where $\mathrm{B} 20=\mathrm{B} 16$ for $(\mathrm{x}, \mathrm{y}, \mathrm{z})$

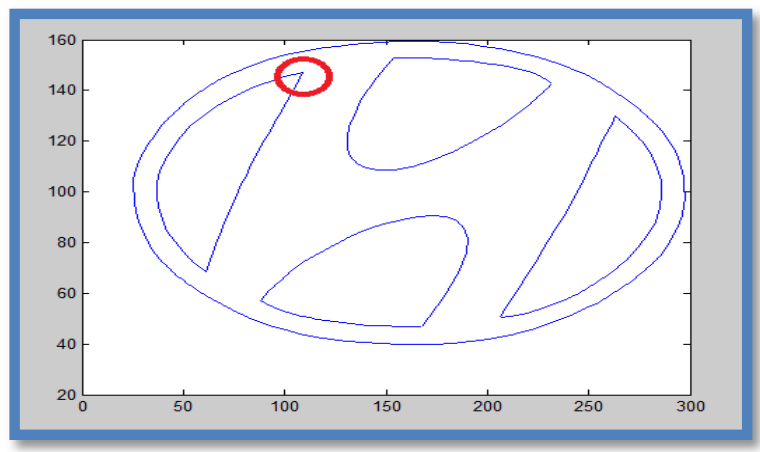

Fig. 5. Continuity example for curves of $\mathrm{C}^{0}$ Bezier curve.

To confirm $\mathrm{C}^{1}$ The equation of the first and next curves should derivative, then substituting (u $=$ one) in first derivative of equation and substituting $(\mathrm{u}=$ zero) in derivative of equation for next curve to find B21 for the next curve as equation below.

$\mathrm{B}^{\prime}(\mathrm{u}=$ one $)$ first curve $=\mathrm{B}^{\prime}(\mathrm{u}=$ zero $)$ next curve as shown in figure (6).

Then B21 =2*B16-B15 for $(\mathrm{x}, \mathrm{y}, \mathrm{z})$

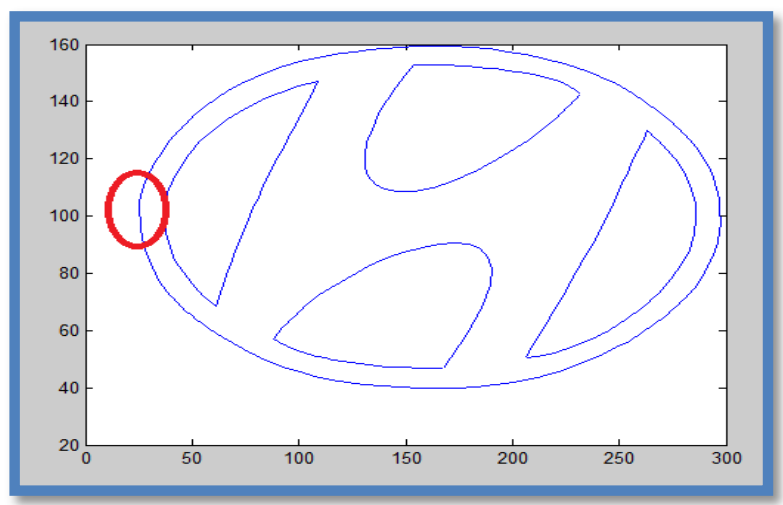

Fig. 6. Continuity for curves of $\mathrm{C}^{1}$ Bezier curve.

And to take $\mathrm{C}^{2}$ the equation of the first and next curves must be second derivative, then substituting $(\mathrm{u}=$ one $)$ in second derivative of equation for first curve and substituting $(\mathrm{u}=$ zero) in second derivative of equation for next curve to take B22 for the next curve as equation below. $\mathrm{B} "(\mathrm{u}=\mathrm{one})$ first curve $=\mathrm{B} "(\mathrm{u}=$ zero) next curve as shown in figure (7).

$\mathrm{B} 22=\mathrm{B} 14-4 * \mathrm{~B} 15+4 * \mathrm{~B} 16$ for $(\mathrm{x}, \mathrm{y}, \mathrm{z})$ 


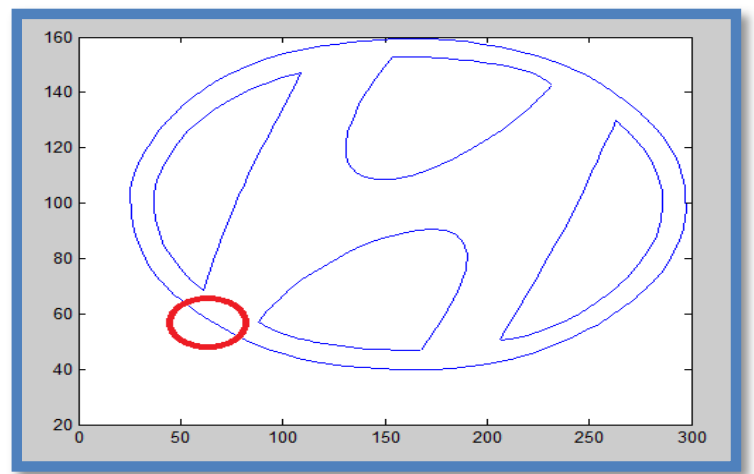

Fig. 7. Continuity example through two curves of $\mathrm{C}^{2}$ Bezier techniques.

\section{Experimental Work}

The experimental work for the proposed case study had been achieved using 3-axis CNC milling machine, where figure (8) explained the final machined case study.

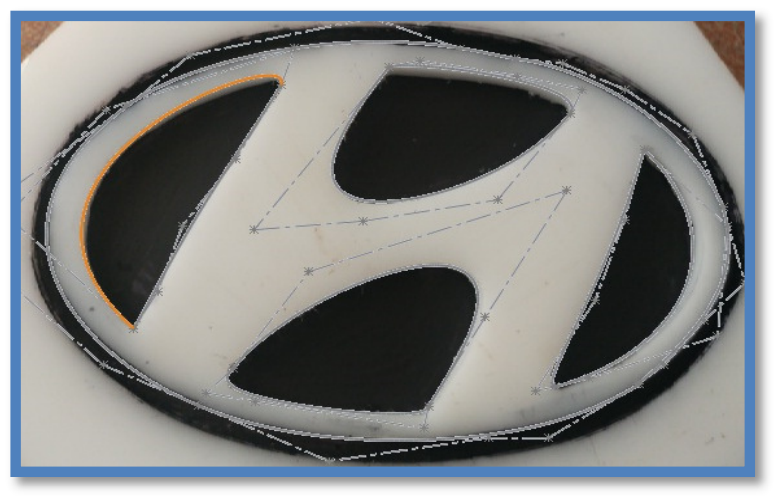

Fig. 8. Final shape of the proposed case study.

\section{Comparison Operation}

Table (1) demonstrates the compression and difference values in dimension between proposed and original part. Where experimental point has been taken from image data while the CAD point has been taken from MATLAB.

Table 1,

The compression and difference values in dimensions between the proposed and original part.

\begin{tabular}{|c|c|c|c|c|c|c|}
\hline No. & $\begin{array}{l}\text { CAD- } \\
\text { POINTS }\end{array}$ & $\begin{array}{l}\text { EXPERMENTAL } \\
\text { POINTS }\end{array}$ & $\begin{array}{l}\text { DEVATION FOR } \\
\text { X-AXIS }\end{array}$ & $\begin{array}{l}\text { CAD- } \\
\text { POINTS }\end{array}$ & $\begin{array}{l}\text { EXPERMENTAL } \\
\text { POINTS }\end{array}$ & $\begin{array}{l}\text { DEVATION } \\
\text { FOR Y-AXIS }\end{array}$ \\
\hline 1 & 153.738 & 153.7 & 0.000388 & 152.938 & 152.098 & 0.008409 \\
\hline 2 & 156.693 & 157 & -0.00306 & 152.918 & 152.098 & 0.008205 \\
\hline 3 & 159.834 & 159.58 & 0.002543 & 152.889 & 152.82 & 0.000696 \\
\hline 4 & 163.183 & 163.002 & 0.001816 & 152.844 & 152.82 & 0.00024 \\
\hline 5 & 166.756 & 166.987 & -0.00231 & 152.773 & 152.77 & $3.71 \mathrm{E}-05$ \\
\hline 6 & 170.557 & 170.503 & 0.000548 & 152.670 & 151.002 & 0.016688 \\
\hline 7 & 174.585 & 174.05 & 0.005354 & 152.527 & 151.002 & 0.015256 \\
\hline 8 & 178.827 & 178.002 & 0.00825 & 152.336 & 151.0036 & 0.013332 \\
\hline 9 & 183.262 & 183.0098 & 0.002523 & 152.091 & 152.008 & 0.00083 \\
\hline 10 & 187.861 & 188.002 & -0.00141 & 151.783 & 152.03 & -0.00247 \\
\hline 11 & 192.585 & 193.0005 & -0.00415 & 151.406 & 152.04 & -0.00633 \\
\hline 12 & 197.389 & 197.889 & -0.005 & 150.954 & 150.999 & -0.00044 \\
\hline 13 & 202.215 & 203.5 & -0.01285 & 150.420 & 151.0008 & -0.0058 \\
\hline 14 & 206.999 & 206.08 & 0.009193 & 149.798 & 149.777 & 0.000217 \\
\hline 15 & 211.668 & 211 & 0.006681 & 149.082 & 149.005 & 0.000776 \\
\hline 16 & 216.139 & 216.058 & 0.000812 & 148.266 & 148.999 & -0.00732 \\
\hline 17 & 220.321 & 222 & -0.01678 & 147.345 & 148 & -0.00655 \\
\hline 18 & 224.115 & 224.105 & 0.000103 & 146.312 & 146 & 0.003124 \\
\hline 19 & 227.411 & 227.41 & $1.83 \mathrm{E}-05$ & 145.163 & 145 & 0.001636 \\
\hline 20 & 230.093 & 232.015 & -0.01921 & 143.893 & 143.025 & 0.008686 \\
\hline 21 & 232.034 & 233.06 & -0.01025 & 142.497 & 142.44 & 0.000576 \\
\hline 22 & 153.738 & 153.548 & 0.001908 & 152.938 & 152.99 & -0.00051 \\
\hline 23 & 149.781 & 148.352 & 0.014294 & 149.329 & 149.33 & $-3.2 \mathrm{E}-06$ \\
\hline 24 & 145.482 & 144.268 & 0.012143 & 145.068 & 145.0325 & 0.000358 \\
\hline 25 & 141.225 & 140.368 & 0.008573 & 140.377 & 141 & -0.00623 \\
\hline
\end{tabular}

\section{Discussion}

1. Implementing error analysis tools to interpret error into meaningful information and give the designer feedback on how well the new surface matches the existing one, by using statistical analysis like average of error, standard deviation, error percentage. Also, 
implementing visual analysis on error to show distribution of error. The compression and difference values in dimensions between the proposed and original part as shown in figure (9 and 10).

2. Machining the reconstructed surfaces using vertical $\mathrm{CNC}$ milling machine and inspect these machined surfaces using image processing to deduce the machining deviation compared with CAD surface model.

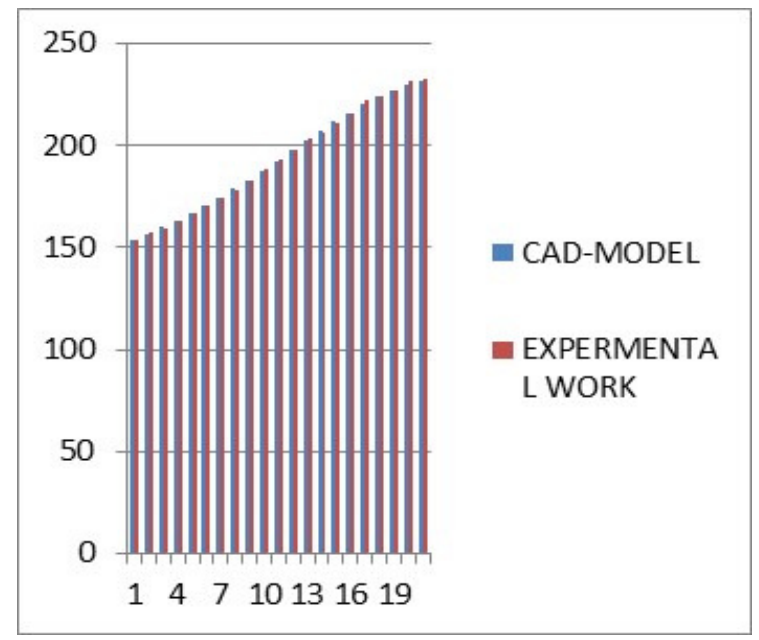

Fig. 9. The compression and difference values in dimensions between the proposed and original part for $\mathrm{X}$-axis.

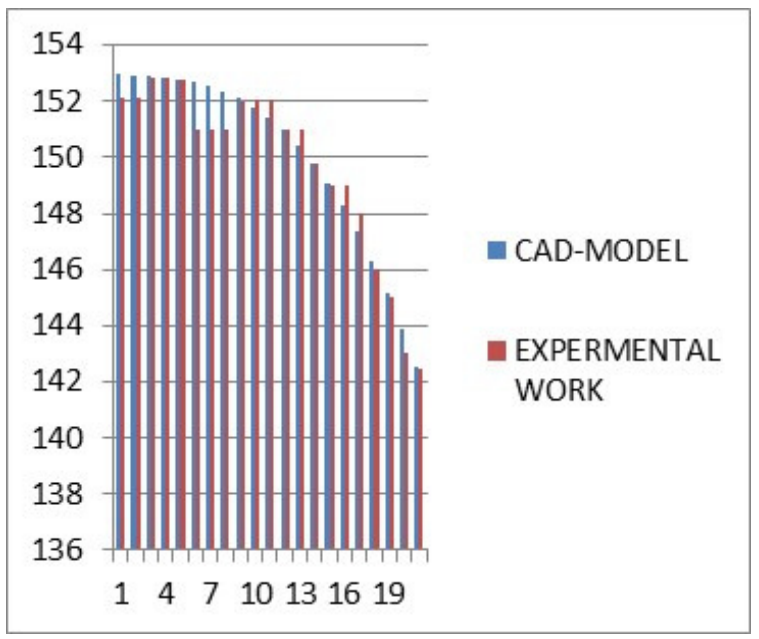

Fig. 10. The compression and difference values in dimensions between the proposed and original part for Y-axis.

\section{Conclusions}

The novel procedure focuses on contraction the point data. The adopted operation for detecting the control points newly has become practical in terms of speed and accuracy, where a great number of point data is being created in the data procuration phase of reverse engineering. Hence, it can be conclude from the compression and difference values in dimensions between the proposed and original part, the success and accuracy of the adopted method based on the deviation in reading that shown in table (1). Finally, this method can be used in reverse engineering depending on the images that taken for the part which wanted to design and manufacture it based on the facilities in the CAD/CAM programs.

\section{References}

[1] B. L. Curless," New Methods for Surface Reconstruction from Range Images", Department of Electrical Engineering, Stanford University, June, 1997.

[2] M. Poniatowska," Deviation Model Based Method of Planning Accuracy Inspection of Free-Form Surfaces Using C.M.M", Division of Production Engineering, Faculty of Mechanical Engineering, Bialystok University of Technology, 2012.

[3] A. Kus, "Implementation of 3D Optical Scanning Technology for Automotive Applications" Sensors 2009, 9, Uludağ University, 2009.

[4] W. Kadhim, M. Mohammed., "Constricting Multi-Patches B-spline Surfaces" Eng. \& Tech. Journal, University of Technology, Vol. 31, Part (A), No. 1, 2015.

[5] D. Zhang, P. Yang and X. Qian, "Adaptive NC Path Generation from Massive Point Data with Bounded Error", Department of Mechanical, Materials and Aerospace Engineering, Illinois Institute of Technology, Journal of Manufacturing Science and Engineering, Vol. 131 / 011001-1, February 2009.

[6] M. Tawfiq, A. abdusamii, S. Kadhim, Ahmed, "enhancement tool path generation using ANN", Eng. \& Tech. Journal, University of Technology, Vol. 31, Part (A), No. 1, 2016. 


\title{
تمثيل الهندسة العكسية عن طريق استخدام المعالجات الصورية
}

\author{
نارين حافظ عبيد***

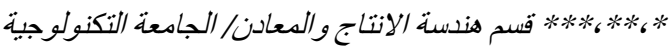 \\ قالبريد الالكتروني: yahoo.co.uk
}

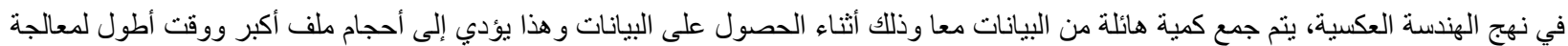

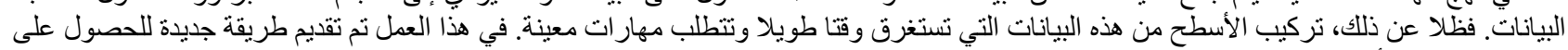

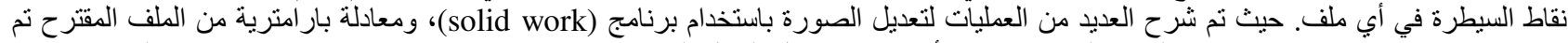

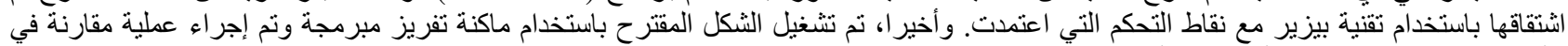

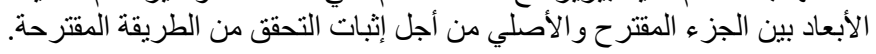

